\title{
Intersectorialidad, interdisciplinariedad y educación en salud: Cuestión de responsabilidad social universitaria
}

\author{
Intersectorality, interdisciplinarity and health education: question of university social responsibility
}

\section{Marina Sánchez-Sanabria', Angélica Romero-Daza², Nancy Hernández-Salas ${ }^{3}$}

1 Mg. en Salud Pública, PhD. en Ciencias de la Educación. Docente Facultad Ciencias de la Salud. Universidad Popular del Cesar. Valledupar, Colombia. e-mail: marinasanchez@unicesar.edu.co

2 Mg. en Enfermería. Docente Facultad Ciencias de la Salud. Universidad Popular del Cesar. Valledupar, Colombia.

e-mail: angelicaromero@unicesar.edu.co

3 Mg. en Enfermería. Docente y Decana Facultad Ciencias de la Salud de la Universidad Popular del Cesar. Valledupar, Colombia. e-mail: nancyhernandez@unicesar.edu.co

Sánchez-Sanabria M, Romero-Daza A, Hernández-Salas N. Intersectorialidad, interdisciplinariedad y educación en salud: Cuestión de responsabilidad social universitaria. Univ. Salud. 2015;17(2): 280 - 289. DOI: http://dx.doi.org/10.22267/rus.151702.12

Artículo de reflexión con el objetivo de señalar el valor de la intersectorialidad, la interdisciplinariedad y la educación en salud como pilares para desarrollar un modelo de responsabilidad social universitario cimentado en la conciencia social y cultura del valor de la otredad y de la alteridad. Surge de los resultados obtenidos de la investigación sobre percepción de riesgos que asumen los habitantes de mayor vulnerabilidad del área urbana de Valledupar con unos estilos de vida que pueden ser modelizados en bienestar saludable y a través del empoderamiento para desarrollar capacidad de autogestión para mejorar las condiciones de vida. El trabajo permite concluir es necesaria una mayor presencia institucional que de manera coordinada potencie los esfuerzos estatales y privados, en donde la universidad tiene un gran compromiso para promover proyectos con pertinencia y conciencia social.

Palabras clave: Acción intersectorial, educación en salud, participación ciudadana. (Fuente: DeCS, Bireme).

\begin{abstract}
The objective of this article of reflection was to point out the value of the intersectorality, interdisciplinarity and health education as pillars to develop a University social responsibility model founded on social awareness and culture of the value of otherness. It comes from the results of the research made about the perception of risks the inhabitants of greater vulnerability of the urban area of Valledupar take who have life styles that can be modelled through empowerment and healthy well-being to develop the capacity for self-management to improve living conditions. It can be concluded that it is required to have a greater institutional presence that enhances State and private efforts, where the University has a strong commitment to promote projects with relevance and social awareness.
\end{abstract}

Keywords: Intersectoral action, health education, citizen participation. (Source DeCS, Bireme) 


\section{Introducción}

Investigar sobre los mayores riesgos contra la salud que asumen las personas en su vida cotidiana, permite al salubrista caracterizar y entender la interacción multifactorial y multidimensional de los procesos salud enfermedad con la mirada holística en la identificación de directrices que fomente la promoción y prevención con programas de educación en salud que empodere a las personas en su autocuidado y cuidado primario, construyendo una cultura saludable y consecuente con la responsabilidad social.

Los estilos y modos de vida son resultados del valor cultural que se hereda, se gesta y se desarrolla en la vida cotidiana de las personas, familias y grupos sociales deben ser comprendidos para modelizar patrones de cuidado, basados en la integración conceptual de las diferentes áreas del conocimiento que converjan en un abordaje integral resultado de una mirada holística y de una postura bioética en su intervención como sustantivo de la responsabilidad social. ${ }_{1-3}$

Tratar la responsabilidad social con espacios dialógicos ciudadanos genera y consensua prácticas éticas socialmente responsables dentro del marco mínimo intransable de los derechos humanos. ${ }_{4,5}$

Precisando así, que la responsabilidad social se obtiene siendo responsables a unos de otros. ${ }_{6}$ En este sentido, las autoras reconocen que en la universidad toda relación debe estar basada en el principio de corresponsabilidad para la construcción de la sociedad que se desea, contemplando el principio individual de responsabilidad, sabiendo que es de ley cumplir los pactos; considerando la convergencia interinstitucional local para dar coherencia a la sociedad en su gestión de cuidado de la salud y de la vida. $_{7-9}$

Conscientes de este principio dentro de la responsabilidad social, la Universidad Popular del Cesar, conformó un equipo interdisciplinar para determinar un modelo de proyección social que visibilizara el impacto de su participación en la solución de problemas sociales; en este sentido se hizo un estudio descriptivo, retrospectivo y transversal, analizando desde la percepción de los habitantes del área urbana de Valledupar, los riesgos que asumen cotidianamente en sectores mayormente vulnerables por su condición socioeconómica determinando el elemento muestral representado en la persona y/o jefe de cada familia, correspondiendo a 372 familias como población total del área urbana, información proporcionada por Emdupar S.A. E.S.P, del censo 2005 y estimada para el año 2013, para una muestra representativa por estratos 1:63, 2: 49 y 3:68 familias ubicadas en las 6 comunas existentes para analizar las variables sociodemográficas, condición de vida, el cuidado de la salud y aspectos higiénico sanitario de expendio de alimentos, que permitieran holísticamente identificar los riesgos que se asumen; derivando lineamientos para focalizar la proyección social por comuna y estrato socioeconómico que sean de mayor vulnerabilidad, que puedan ser modelizados desde la educación para la salud y de aquellos que empoderando a la comunidad pudiesen crear corresponsabilidad para asumir el control social requerido para la gestión en salud, destacándose la necesidad de hacer esfuerzos mancomunados inter estamentariamente, trabajando por consenso entre programas académicos para establecer programas de desarrollo comunitario. $_{10}$

El modelo de proyección social para la Facultad Ciencias de la Salud de la Universidad Popular del Cesar fue liderar el trabajo en la comuna cuatro en los barrios de estrato socioeconómico dos, desarrollara programas de proyección social con trabajo interdisciplinar e interinstitucional con los siguientes considerandos: $_{11}$

\section{La corresponsabilidad, valor bioético en la responsabilidad social universitaria}

Gestionar la ética organizativa - empresarial, considerando la universidad como agente moral que forma para desarrollar en los egresados capacidad para valorar en los procesos públicos exige enseñar con el ejemplo, y es a partir de esta premisa en la que se afirma que le compete al docente liderar esa pertinencia, pero al mismo tiempo que la universidad, como las demás instituciones locales, den condiciones para su favorabilidad. ${ }_{12}$

El desarrollo del liderazgo ético reconoce la capacidad del ser humano para actuar por motivos trascendentes en beneficio del progreso colectivo, requiriéndose en el trabajo en equipo, la capacidad de 
consensuar con todas las personas involucradas en el cambio esperado y proyectado, fundamentado en una visión holística del trabajo, un sentido de comunidad y un proceso de toma de decisiones compartido, dejando el protagonismo y anteponiendo como principal valor el servicio a los demás, destacando la importancia interinstitucional en la mejora de las condiciones de vida de las personas, así como, en el progreso de la sociedad. $_{13}$

La responsabilidad social se asume a través de acciones que protegen y mejoran el bienestar de la sociedad a medida que trata de alcanzar sus propios objetivos, es la forma en cómo afecta a la comunidad; significa que para la Universidad Popular del Cesar, ser socialmente responsable, debe incluir objetivos sociales desde los procesos de planeación, visibilizar normas de funcionamiento integrado y complementarios e informar cotidianamente en todas las instancias, los progresos y experimentar diferentes métodos para medir el desempeño social; cultura que proyecta reputación corporativa, promueve admiración, valoración y confianza que incentiva credibilidad para el posicionamiento social; asegurando su sostenibilidad a través de su práctica social en la interacción armónica docencia, investigación y proyección social con fundamentaciones éticas, para que sea auténtica. ${ }_{13-16}$

Si la ética establece, en los distintos escenarios de la universidad, los principios que definen sus actuaciones, entonces le corresponde a la responsabilidad social preocuparse de las consecuencias que esas actuaciones puedan tener sobre la sociedad en general o en ciertos grupos en particular; significa que cada actuación sujeta de cada misión de la universidad deba repensarse, involucrando en cada proceso a los potenciales

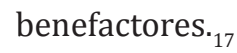

Ser socialmente responsable significa ir más allá de su cumplimiento, avanzando voluntariamente hacia nuevas exigencias, mayor inversión en capital humano, mejor relación con los interlocutores, mayor respeto al medio ambiente. ${ }_{18}$ El conjunto de valores, normas y principios que se evidencia en la cultura organizacional en la universidad facilitará una mayor influencia en la sociedad permitiendo una mejor adaptación a diversos entornos al respetar sus derechos y los valores compartidos. ${ }_{19}$
La universidad como centro de producción y construcción del conocimiento, requiere que las decisiones de sus actores generalmente dados desde la racionalidad técnica y la lógica, asuman como episteme, la complementariedad con la comprensión de los fenómenos para la mirada holística de los problemas sociales y hacer unísona la relación sociedad- tecnología - sociedad y no seguir actuando con vicios mecanicistas, guiados por el deber ser de lo que prescribe la normativa tradicional, que no va a tono con los avances de un desarrollo pertinente de la localidad y que en la mayoría de los casos hacen inflexibles los procesos de toma de decisiones, dificultando la comunicación y participación voluntaria mediante el diálogo y acuerdos inter estamentarios y proactivos; ${ }_{19,20}$ códigos de ética que deben ser asumidos en la toma de decisiones, en los procesos y objetivos estratégicos para mejorar el clima organizacional.

La razón de ser de los códigos de ética es proveer una guía de conducta moral de las personas y dar legitimidad y soporte a las decisiones, de tal manera que a la Facultad Ciencias de la Salud le corresponde asumir con responsabilidad su misión con la sociedad; y el modo en cómo está respondiendo a esas expectativas implica que desde la bioética debe estar al servicio de la sociedad y cubrir los desafíos inaplazables que ésta le plantea, en correspondencia con los resultados del estudio de riesgos que enfrentan los habitantes del área urbana de Valledupar; cuyo valor agregado, obliga a contextualizar el currículo, mantener como herramienta la actualización epidemiológica de la situación salud enfermedad y de la comprensión de los fenómenos; identificando las poblaciones que tienen mayor riesgo, en donde se requiere del acompañamiento y liderazgo de los programas académicos en salud y otros afines al control higiénico sanitario (Ingeniería Sanitaria) y Promoción de la Salud. ${ }_{20,21,10}$

El docente por tanto, deberá identificar y reconocer las características del contexto en el que desarrolla su intervención educativa, utilizando como principal herramienta la reflexión de la práctica pedagógica, comprendiendo que la realidad influye en el individuo, pero que el individuo también tiene la posibilidad de actuar sobre ésta con el fin de transformarla para mejorarla, por ello, el valor agregado de una pedagogía transformadora se revierte en una 
fortaleza para la calidad de vida de las familias de los estudiantes y a la comunidad perteneciente. ${ }_{22-24}$

\section{Intersectoraliedad, interdisciplinariedad, educación en salud, trabajo en equipo: eje horizontal del modelo de responsabilidad social universitaria}

Con los resultados encontrados en la investigación mencionada anteriormente, se ilustra qué aspectos de las variables condujeron a las autoras a determinar que la intersectorialidad, interdisciplinariedad, educación en salud, trabajo en equipo fuesen los ejes horizontales del modelo de Proyección Social Universitaria:

\subsection{Situación sociodemográfica}

Desde los 26 años de edad, la población alcanza sólo $32,5 \%$ la formación de bachillerato, 24,3\% educación superior; una ocupación del 73,8\% con mano de obra no calificada, trabajo independiente, oficios varios, vendedores o en la mendicidad y el trabajo doméstico en mujeres, quienes forman pareja a temprana edad y en unión libre.

El 46,1\% de hogares trabajan como mínimo dos personas por familia, por cada 4 hogares de jefatura masculina, un hogar es con jefatura femenina $(303 / 68)$, las mujeres alcanzan la primaria como máximo grado de formación, reconociendo que los ingresos no alcanzan cubrir necesidades básicas, ni recibir beneficios de políticas públicas si son cabeza de hogar. El 4,3\% de estas familias tienen menores trabajando, sobre todo en comuna 4; estrato 2.

EL 69,3\% reconoce las Juntas de Acción Comunal, $80,9 \%$ no asisten a reuniones, justificando en su mayoría por falta de tiempo o porque no funcionan; estrato 2 , comuna 4 .

El 87,9\% reconocen la inseguridad como mayor problema, seguido de la prostitución, drogadicción y vandalismo, especialmente el estrato 2 , comuna 4.

Reciben intervención policial cuando la inseguridad es por vandalismo en un $53,7 \%$ seguida de la violencia intrafamiliar en un $28,2 \%$, seguida de muerte violenta el 27,2\%, reconocen mayor consumo de sustancias psicoactivas por alucinógenos, seguidos del bóxer. Comuna 4.
Aspectos anteriores que evidencian la ausencia institucional para promover ocio productivo, fomentar cultura ciudadana, vigilancia epidemiológica, control sanitario de expendios, mantenimiento de vías, parques, redes eléctricas, fomentar la convivencia pacífica e intercultural, ampliar coberturas de servicios de salud con calidad y centren la condición humana en sus procesos de atención, desarrollo de políticas de formación para el trabajo y control y manejo de basuras.

No hay procesos de ciudadanía afectando los procesos de democracia. Las familias reconocen la labor de la Juntas de Acción Comunal, pero no participan, aspecto que imposibilita mejorar sus condiciones de vida dentro de la comunidad, además se niegan la oportunidad de desarrollar proyectos colectivos, individuales, grupales y la realización de obras de gestión social.

La población joven no está accediendo a una formación académica que les cualifique en su proyección ocupacional, quienes sólo alcanzan el último grado escolar de educación media a cambio de buscar recursos económicos para el sustento de sus familias; de ahí que la mayor ocupación de la población es el fenómeno del rebusque y el ocio canalizado para juegos de azar, consumo de licor, droga, prostitución; escenario propicio para la violencia en todos los ámbitos de la vida humana; siendo un problema social que fue reconocido en el estudio y que alteran procesos de convivencia pacífica y de salud mental comunitaria.

Cada uno de los riesgos descritos deja entrever la ausencia o débil planeación de abordaje intersectorial, interdisciplinar, educación en salud y trabajo en equipo, cuando la convergencia de diferentes disciplinas y coordinación para la atención a los problemas sociales, facilita una mirada holística y plantea mejor el proceso de acompañamiento a los benefactores. $_{25,26}$

\subsection{Condiciones de vida}

La calidad de vida de una persona es dada en primer término por la posibilidad devivir demanera agradable con el entorno, que de contarse con la mayoría de los recursos materiales contribuyen a la satisfacción, y por ende, son ingredientes motivacionales que junto a la personalidad de cada individuo van determinando 
estilos de vida, evidenciados en sus prácticas sociales y en su relación con el entorno ambiental ${ }_{27,28}$

El estudio de referencia, visibiliza la falta oportunidad y accesibilidad a mejores condiciones de vida en el ámbito cultural, laboral, ambiental, servicios públicos con deficiencia sanitaria en expendios de alimentos existentes en la comunidad según líderes comunitarios, con los siguientes riesgos: El 25,9\% de las familias manifiestan que realizan actividades deportivas, el $57 \%$ de la muestra afirma que el tiempo de ocio en hombres, es dedicado a juegos de azar y de mesa, y el de las mujeres a actividades religiosas; no cuentan con transporte público óptimo ni suficiente, que convierte a la actividad del mototaxismo en una oportunidad de mayor ocupación (100\% de uso) y es reconocida como la causa de mayor contaminación atmosférica y medio para propiciar atracos y violación, según lo manifestado por los informantes quienes afirman que uno de los mayores riesgos son los atracos $(62 \%)$.

El 23,8\% reconoce que la comunidad es quien hace el mantenimiento de las vías, el 14,2\% expresa que tienen alcantarillado y el $76 \%$ dice que no tienen rejillas. Fenómeno que está muy relacionado con la delincuencia según lo manifiesta los habitantes; el $70 \%$ reconocen que los espacios públicos no funcionan y el $57 \%$ de ellos dice que les falta mantenimiento, aunque reconocen que el Estado lo realiza, no es suficiente y el $62 \%$ afirma que los lotes baldíos son un espacio de violencia.

El 37\% de las familias no tiene vivienda propia sobre todo en el estrato 1 comuna 4. Manifiestan tener conexiones ilegales de redes eléctricas, el $14 \%$ a nivel domiciliarios y un $9,5 \%$ en espacios públicos, además no cuentan con mantenimiento, aspecto relevante por sus implicaciones y consecuencias a gran escala.

Faltan proyectos colectivos y sociales liderados desde la intersectoraliedad, capacitando y organizando todo un plan de atención necesaria, fortalecer redes de mercado apoyado desde la academia para fomentar la protección del menor trabajador, a la mujer por la violencia misma y ausencia de proyectos de vida; y proponer alternativas de solución a los deficientes medios de transporte y a la calidad del servicio.
Estas condiciones que aumentan los eslabones de pobreza y destrucción de la vida del planeta como resultado de los mecanismos de sobrevivencia al cual recurren y que caracterizan la vida cotidiana de los estratos socioeconómicos bajos; implicados dentro de una cultura dominante en una sociedad de consumo, trae consigo analfabetismo, desigualdad social, desnutrición y migración.

Lograr comprender desde la universidad, las diversas expresiones de la pobreza, adentrarse en su significado y tratar de efectuar algo al respecto, es el único camino opcional de cortar con el círculo vicioso de justificar en lo público las debilidades sociales y económicas si se tiene conciencia social y corresponsabilidad, fomentando valores y responsabilidades, reencausando la epistemología de la proyección social y extensión, desarrollando programas de educación que revierta en la salud de los estudiantes y de la comunidad local, empoderándolos para la autogestión de su desarrollo a través de la generación, enseñabilidad, transferencia, aplicación y humanización del conocimiento. $29-31$

\subsection{Cuidado de la salud}

Son las mujeres a quienes la sociedad le ha delegado la función de responder con la crianza de los hijos para la sociedad y contemporáneamente, incursiona en la generación de ingresos para el sostenimiento de la familia; convirtiéndose en una política de protección en todas las sociedades del mundo, al contar con generaciones modernas que han favorecido cambios en el estilo de vida en las diversas naciones por su rol, características y condición humana, que ha favorecido la forma de cómo construyen el qué y el cómo del cuidado de su salud y la de su familia; asumiendo con responsabilidad las demás obligaciones y deberes como mujer, persona, madre, compañera, profesional y trabajadora. ${ }_{32-34}$

Las rutinas cotidianas e interacciones durante el cuidado, al ser estudiadas por el salubrista, le permite caracterizarlas y analizarlas para determinar la red presente en los patrones de cuidado que forman parte de una medicina doméstica. ${ }_{34}$

Al respecto en el estudio en referencia, se encontró que: ${ }_{11}$ El $45 \%$ se automedica buscando respuesta oportuna en droguerías, el $56,5 \%$ con lo que hacen al alcance dentro de su propia casa, por barreras 
socioeconómicas en un $25 \%$ o por la falta de acceso a servicios de salud; situación que agudiza su condición de vulnerabilidad; siendo más evidente en el estrato 1 de la comuna 4.

Al programa prenatal accede un $95,2 \%$ de las gestantes; el 43,9 \% de mujeres en edad fértil presentan esquema de vacunación incompleto; el $31 \%$ de las inscritas en el programa de planificación familiar, adoptan el método anticonceptivo hormonal; el $10,8 \%$ de mujeres con vida sexual activa no se realiza citología y el $40 \%$ no se realiza el autoexamen de mama; situación observada con mayor frecuencia en las comunas 3,4 y 5 .

El 74,3\% manifiesta que la población adulto mayor no se ha realizado examen de próstata en el último año y que el $63,2 \%$ ha presentado enfermedades cardiovasculares y que el $79,2 \%$ de ellos reciben tratamiento médico. El 13\% hacen cepillado dental al menos una vez al día y un $11 \%$ de 152 adultos mayores han tenido control odontológico hace más de un año, el resto en menos tiempo. Existe presencia de discapacidad en el $6.7 \%$ de los niños, relacionada con la conducta, lenguaje, motor, visual y auditivo.

El $62,2 \%$ de las mujeres ofrece lactancia materna hasta los 6 meses de edad cuando inician la alimentación complementaria; en tanto que el 25.9\% ofrecen lactancia materna por periodos mayores a un año.

El 21\% de las familias reconocen quelas enfermedades respiratorias son más frecuentes en menores de cinco años; el 9,4\% de 203 menores de 10 años, no asisten al programa de crecimiento y desarrollo.

Con relación al saneamiento básico y medio ambiente existe convivencia con micos, aves, gatos y perros, sin vacunar en su gran mayoría, con disposición final de heces a campo abierto.

Los mayores riesgos percibidos por las familias en este campo son la humedad excesiva 15,5\% e inundaciones $7,7 \%$, pese a esta percepción no se vinculan a una institución para la atención de desastres, ni ejercen un voluntariado.

El 33\% expresa que el servicio de urgencia de la IPS más cercana no tiene recurso humano básico para la atención necesaria y para atender el servicio de ambulancia (43\%). Se evidencia por tanto:

-Deficiencia en el cuidado primario a nivel de las familias, en la atención al niño, al adolescente, a la mujer, al adulto mayor, en la salud mental de la familia, en el manejo sanitario de la vivienda y en el manejo del entorno natural.

-Deficientes medidas de autocuidado y de programas de uso de programas de promoción y prevención, falta educación para la salud priorizados según problemática descrita.

Son pocas las familias que reconocen programas de educación sobre temas que son de vital importancia para el cuidado de la salud y la enfermedad, situación limitante para el afrontamiento de tema y problemas de interés común entre habitantes del sector, coadyuvado por la falta de compromiso frente a la participación comunitaria.

La crianza y la educación pertinentes tienen un impacto positivo en el desarrollo de actitudes saludables y democráticas posteriores, pero también de amor a sí mismo y por ende a los demás para afrontar en forma efectiva las exigencias y desafíos de la vida diaria con bases cimentadas para el desarrollo psicosocial del bebé y su futura salud mental, desde las primeras relaciones o vínculos afectivos. ${ }_{28,35}$

La importancia de estos temas radica no sólo en el valor que se le concede en las políticas de salud o a las necesidades básicas insatisfechas o a la violación de los derechos, sino que los esfuerzos no siempre se expresan en una reducción efectiva en las estadísticas de salud, que dado en el juicio de expertos, responden a cuáles han sido los obstáculos para lograr la salud para todos. $_{36,37}$

En los desastres naturales, las comunidades que sufren los mínimos estragos y que se recuperan con mayor celeridad son aquellas que pueden confiar en sistemas de apoyo mutuo y en recursos propios; en este sentido le compete a la universidad liderar procesos de organización comunitaria y educación masiva en primeros auxilios y coordinar con la Cruz Roja, Defensa Civil, para lo pertinente. ${ }_{38}$ 


\subsection{Condiciones higiénico sanitaria de expendio y centros de consumo de alimentos}

Los diversos expendios de manejo de alimentos considerados (tiendas, restaurantes, expendios de lácteos y carnes) no cumplen o no aplican las medidas higiénico sanitario en lo que respecta al área locativa y la mayor presencia del riesgo está en la mala calidad de las superficies de contacto directo con el alimento que no poseen un acabado liso, y están porosos; dando cabida a toda una gestación de cadena epidemiológica anti sanitaria en este sentido.

Igualmente, se caracterizan por el incumplimiento en el manejo de limpieza y control de basuras, insuficiente iluminación natural y/o artificial en todos los estratos, sin ventilación adecuada, falta capacitación en buenas prácticas de manejo de alimentos y control del personal mediante exámenes periódicos.

La apertura de negocios emergentes está relacionada con las necesidades económicas de las familias, pero sin un grado de responsabilidad social, por el riesgo que representa y sus efectos a gran escala, tanto para ellos como para los consumidores o clientes. Si es esta la situación de los centros de servicio con área locativa, los investigadores suponen más grave aún el de aquellos negocios clandestinos y de preparación de alimentos.

\section{Reflexión}

Entre mayor pobreza exista, mayor es el riesgo relativo para el cuidado de la vida,al estar sujeto por sistema social de valores, creencias y valores culturales; aportando a la causa individual más importante de la miseria humana en el mundo actual; en especial si se sigue atribuyendo la pobreza a causas falsas, pues se observa que existe una correlación entre niveles educativos obtenidos y la profesión u ocupación que desarrollan en el presente. ${ }_{38}$

Si no se rechazan las actitudes clásicas del trabajo arduo, el ahorro, la cooperación, y la iniciativa propia, y en su lugar, hacen responsables por el progreso al gobierno, a los sindicatos y a organismos internacionales y sus dádivas, de las cuales en gran parte es responsable el mismo gobierno con sus programas sociales que se convierten en paternalistas perpetuando la cultura de mendicidad y el bajo nivel de respuesta de la comunidad en su autocuidado ${ }_{39,40}$ La uniformidad cultural resultado de la globalización de la economía, basado en el consumismo y de una economía controlada por fuerzas mundiales excluyentes y contrarias a la emancipación social, no parece ser un escenario deseable, por el desconocimiento de lo local; de ahí que debe fomentarse la participación comunitaria con integrantes conocedores y sensibles de sus propios problemas; crear estos ambientes saludables para personas de todas las edades, será necesario contar siempre con su participación activa en los esfuerzos de las organizaciones de base, los ciudadanos particulares, los líderes de la comunidad, los profesionales de la salud, docentes e investigadores, trabajar en forma conjunta para lograr los cambios que permitan el progreso de estos espacios. ${ }_{40,45}$

La vinculación comunitaria en la proyección social universitaria es una mezcla entre ciencia y arte, porque se fortalece de varios saberes del área socio humanístico; los conceptos de organización se obtienen de la literatura sobre la participación comunitaria, movilización comunitaria, creación de grupos de interés, psicología comunitaria y del valor de la cultura; y del arte aplica y adapta la ciencia en formas que se ajusten a la comunidad de interés y a los objetivos de los esfuerzos específicos de vinculación comunitaria para gestionar en el aula los procesos de enseñanza y aprendizaje; de tal modo que los resultados de estos esfuerzos pueden definirse de manera distinta y pueden abarcar una amplia gama de estructuras como coaliciones, asociaciones, colaboraciones, pero todas quedan comprendidas dentro del concepto general de vinculación comunitaria; así, la universidad le apuntará a la construcción de ciudadanía y a la cultura del cuidado de la vida $_{46,47}$

Por tanto, es necesaria la interdisciplinariedad para trabajar en los diversos grupos sociales y las familia puesto que desde el objeto disciplinar de cada profesión, aportarán en consenso una corresponsabilidad pertinente para la gestión del cuidado de la vida. ${ }_{48-50}$

Frente a la tendencia homogeneizadora, las culturas locales encuentran un buen aliado en el diálogo con las identidades de forma flexible, múltiple 
y abierta; le compete a la universidad fomentar su empoderamiento para lograr un compromiso ciudadano por la conciencia social y de su valor dentro de una cultura saludable.

Las directrices de un modelo de responsabilidad social universitaria deben centrar la interdisciplinariedad e intersectorialidad y el trabajo en equipo, toda vez que se pretenda abordar integralmente los problemas sociales con alternativas de solución basadas en la corresponsabilidad, siempre y cuando haya coherencia proyectiva en la misión interinstitucional y de una construcción de cultura ciudadana por el valor de la vida y la salud basada en el esfuerzo colectivo y cimentada en el consenso.

\section{Conclusión}

Una comunidad con problemas sociales, que dejan su formación académica, asumen trabajos de mano de obra no calificada y no pertenecen a organizaciones comunitarias, se vuelve más vulnerable, pues sus actitudes y estilos de vida marcan las aspiraciones y formas de administrar la vida; exige una mayor presencia institucional de manera coordinada para potenciar los esfuerzos estatales y privados, que de manera complementaria busquen el bien colectivo, correspondiéndole a la universidad asumir el rol formador y de liderazgo interinstitucional pertinente a una sociedad que requiere del aprendizaje y de la enseñanza para la generación de nuevos conocimientos, mayor sensibilidad social y emprendimiento, para optimizar los esfuerzos e inversiones estatales, que en ocasiones son incordinadas e infructuosas, porque no obedecen a las necesidades de la comunidad, no son sólidas, claras, ni transparentes.

\section{Agradecimientos}

Al cuerpo administrativo - académico de la universidad y especialmente a la Facultad Ciencias de la Salud por su disposición para apoyar al grupo de docentes en la determinación de directrices para la propuesta de proyección social soportadas contextualmente y científicamente. A los docentes y estudiantes de las diversas disciplinas y a la comunidad en general, por su participación en las diferentes fases del trabajo.

\section{Referencias}

1. Sánchez SM. La cultura como valor en la promoción y prevención: Un reto para la formación de enfermería; 2009. En: saberes culturales en un mundo glocalizado. www.index-f.com/para/n7/sumario.php

2. Ruelas-B E. La importancia de la intersectorialidad en los determinantes de la salud. En: La importancia de los determinantes sociales de la salud en las políticas públicas. Instituto Nacional de Salud Pública. de México 2012:95-97. Disponible en: http://www.anmm.org. mx/lidsspp/pdf/95_pdfsam_la.pdf

3. Cortina CA. La dimensión pública de las éticas aplicadas. Revista Iberoamericana de Educación 2002: 29: 10.España.

4. Cortina CA. Pobreza y libertad. Erradicar la pobreza desde el enfoque de Amartya Sen. Tecnos; 2009.

5. Cortina CA. Neuroética y neuropolítica: sugerencias para la educación moral. Madrid, Tecnos; 2011

6. Ellacuria I, El compromiso político de la filosofía en América Latina. Santafé de Bogotá (Colombia): Editorial El Búho; 1994.

7. Pérez T. La corresponsabilidad de los docentes con los fines de la educación. En: Boletín 829 REDIPE. La evaluación ante los nuevos paradigmas educativos: retos y desafíos para el docente. 2013.

8. Sánchez M, Rondón B. La diversidad cultural en los procesos de formación académica de enfermería requiere el manejo de la ética pedagógica, la corresponsabilidad y un pensamiento mediador. Revista Enfermería Global; 2013.

9. Cortina A. De los derechos a las responsabilidades, del contrato al reconocimiento http://www.aafi.filosofia. net/ALFA/alfa8/alfa8a.htm

10. Sánchez M, Hernández N, Romero A, Jácome. Directrices de un modelo de atención a riesgos trazadores en el cuidado de la vida y la salud a tono con la responsabilidad social de la Universidad Popular del Cesar. Universidad Popular del Cesar; 2013.

11. Sánchez M, Hernández N, Romero A, Jácome J. Responsabilidad social universitaria para el cuidado de la salud de los habitantes área urbana de Valledupar. Universidad Popular del Cesar. Ediciones UNICESAR; 2013.

12. Fernández J. ¿Por qué y cómo gestionar la ética organizativo-empresarial? En: Bajo Sanjuán, Anna y Villagra García, Nuria (eds.); 2007

13. García D. La apuesta ética en las organizaciones sanitarias. Editorial publicaciones de la Universitat Jaume I. Valencia. 2005 
14. Navarro F. Responsabilidad social corporativa. Teoría y práctica en la empresa. Editorial ESIC. Madrid; 2009.

15. Martin de Castro G. Reputación empresarial y ventaja competitiva. Editorial ESIC. Madrid; 2008.

16. Hernández J, Seijo C, Vera C. Rasgos éticos de la gerencia: Posturas sociales para la construcción del saber. Editorial ed: Universidad Popular Del Cesar, Valledupar; 2013.

17. Bernal M, Rodolfo N. Hacia una reflexión ética en la universidad. Universidad de Boyacá; 2003

18. Sánchez M. La diversidad cultural en la formación de enfermeras. Fondo de Publicaciones Universidad Popular del Cesar; 2013.

19. Fernández JL. Ética, responsabilidad social y modelos de empresa. Universidad Pontificia Comillas de Madrid. Disponible en: http://www.eticaed.org/ tica\%20Responsabilidad\%20Social\%20\%20y\%20 Modelos\%20de\%20Empresa1.pdf

20. Alvarado M, Rangel O. Ética: directriz para toma de decisiones en universidades. Revista Guillermo de Ockham. 2012;10(2):65-74.

21. Barragán J. Responsabilidad social empresarial: teorías y experiencias. Caracas: Velea; 2008.

22. Balda RC. Dinámica de la apropiación multicultural del contenido en la formación contextualizada del profesional. Tesis presentada en opción al grado científico de Doctor en Ciencias Pedagógicas. Centro de Estudios Manuel F. Gran, Santiago de Cuba, Cuba; 2009.

23. Mendoza F. Responsabilidad social de las Facultades de Educación y de las Escuelas Normales Superiores en la formación de maestros contextualizados. Simposio: educación, formación docente y práctica pedagógica. Universidad Simón Bolívar; 2014.

24. Calvo MC. Práctica pedagógica, aprendizaje y complejidad de la formación en contexto". Universidad de La Serena, Chile. En Simposio: Educación, formación docente y práctica pedagógica. Universidad Simón Bolívar. 2014.

25. Rodríguez CA. Estrategias docentes interdisciplinares para el abordaje de saberes profesionales en el Área Económico Administrativa. Universidad Juárez Autónoma de Tabasco, Universidad Veracruzana, México. RED INNOVA CESAL. En: http:// www.innovacesal.org/micrositio_redic_2014/ redic_2014_3_rep_gpal_ecoadm.pdf

26. Carvajal EY. Interdisciplinariedad: desafío para la educación superior y la investigación. Rev. Luna Azul. 2010;31:156-16.
27. Andrews FM, Withey SB. Social Indicators of WellBeing. En García Martín, M. A. Desde el concepto de felicidad al abordaje de las variables implicadas en el bienestar subjetivo: un análisis conceptual. Revista Digital. 2006; 8(48). Disponible en: http://www. efdeportes

28. Núñez AC, Tobón S, Arias D. Calidad de vida, salud y factores psicológicos asociados.2010. Rev. Perspectivas en Psicología. 2010;13: 11-32

29. Villareal E. Estrategia para superar la pobreza moderada: Una propuesta de acción interinstitucional. México: Oficinas para las Políticas Públicas, Presidencia de la República. 2003.

30. The world bank. Poverty reduction and growth: virtuous and vicious circles. Washington DC. 2006.

31. Vallaeys F. Responsabilidad universitaria: hacia una definición madura del concepto. Universidad Católica del Perú- Dirección Académica de Proyección Social y Extensión Universitaria. Lima, Perú. 2006.

32. Centro de Investigación, Proyección y Desarrollo. Área: Transferencia y comunicación del conocimiento. Dimensión: Transferencia del conocimiento derivada de la acumulación de saberes de la docencia e investigación Universidad Católica de Manizales; 2011.

33. Gutiérrez Bl. La mujer como agente de cambio en la educación: un estudio de caso. Ponencia presentada en el "Primer Encuentro Estatal de Investigación Educativa".En: La tarea. Rev. de Educación y Cultura Guadalajara, Jalisco; 1995.

34. Zolla C, Carrillo AM. Mujeres, saberes médicos e institucionalización. In: Figueroa JG. La condición de la mujer en el espacio de salud. México (DF): Colegio de México; 1998. p. 167-198.

35. Menéndez E. Grupo doméstico y proceso salud/ enfermedad/atención: del teoricismo al movimiento continuo. Cuad Méd Soc 1992; 59:3 - 18.

36. Tizón J. Funciones psicosociales de la familia y cuidados tempranos de la infancia, ponencia presentada en el II Congreso de la Red Española de Política Social. Madrid; 2010. Disponible en http://www.espanetspain.net/ congreso2010/paneles/panel6

37. Alfonso AC. Salud sexualy reproductiva desde la mirada de las mujeres. Centro Nacional de Educación Sexual. Disponible en: http://www.bvs.sld.cu/revistas/spu/ vol32_1_06/spu10106.htm

38. Tova M, Godinot M, Velasco O. La participación comunitaria en el manejo de desastres: Reflexiones sobre experiencias recientes en Honduras y Nicaragua. Guatemala. Disponible en: http://cidbimena.desastres. hn/RIDH/pdf/doch0115/pdf/doch0115.pdf 
39. Gómez L, Suárez O. Habilidades para la vida. Disponible en: http://www.udea.edu.co/portal/page/portal/ bibliotecaSedesDependencias/unidadesAcademicas/ FacultadMedicina/BilbiotecaDiseno/Archivos / PublicacionesMedios/BoletinPrincipioActivo/85_ habilidades_para_la_vida.pdf

40. Organización Mundial de la Salud. Informe sobre la salud en el mundo 2008 La Atención Primaria de Salud más necesaria que nunca. En http://www.who.int/ whr/2008/08_report_es.pdf

41. Keckeissen E. Las causas de la pobreza en el tercer mundo. Rev. Contribuciones a la economía de la economía de mercado, virtudes e inconvenientes. 2001. disponible en: http://www.eumed.net/ cursecon/colaboraciones/ index.htm

42. Dorantes C. Sociología de la pobreza: El caso de la mendicidad en Querétaro. Universidad Autónoma de Querétaro; 1. edición. Serie Humanidades. Colección Sociología; 1991.

43. Espinosa GL. Cambios del modo y estilo de vida; su influencia en el proceso salud-enfermedad. 2004. En; Rev Cubana Estomatol. 2004;41(3).

44. Leandro G. Pobreza: Conceptos y medición [Internet]. Aula de ecomnomía [cited 2015 Feb 17]. Disponible en: http://www.auladeeconomia.com/articulos18.htm
45. UNESCO. I Foro Unesco de negocios sobre la empresa, el desarrollo humano y la cultura en la edad de la mundialización. (Conferencia intergubernamental sobre políticas culturales para el desarrollo. Estocolmo; 1998.

46. Comité de Función Clave de Vinculación Comunitaria. Principios de vinculación comunitaria 2011. Segunda edición. En: http://www.atsdr.cdc.gov/ communityengagement/pdf/Principles_Community_ Engagement_2ndEdition_Spanish.pdf

47. Villalobos FX. Reflexión en torno a la gestión de aula y a la mejora en los procesos de enseñanza y aprendizajes. Revista Iberoamericana de Educación / Revista Iberoamericana de Educação. 2011.

48. Plan Nacional Decenal de Educación 2006-2016. Referencias a la familia como instancia educativa corresponsable. En: http://www.plandecenal.edu.co/ html/1726/articles-166057_familia.pdf

49. Torres J. ¿De qué hablamos en las aulas?2014. En: http://jurjotorres.com/?tag=interdisciplinariedad

50. Waldow B. El proceso de cuidar según la perspectiva de vulnerabilidad. Rev Latinoam enfermagen. 2008;16(4):1-8. 